VOL. 47 (1993) [521-522]

\title{
Modelling host regulation of Trichostrongylus colubriformis a nematode parasite of sheep
}

\section{ROBERT John Dobson}

Trichostrongylus colubriformis is a species of helminth parasite infecting the alimentary tract of domestic livestock. For this parasite the initial manifestation of immunological control is to prevent a proportion of incoming larvae from establishing. As host immunity develops, a smaller proportion of infective larvae establish themselves and eventually adult parasites will be rejected by the host. It is this host-parasite interaction which this study examines in detail, so that mathematical models can be formulated to describe the resistance status of the host and the dynamics of its worm burden.

Factors influencing the regulation of $T$. colubriformis populations within the host were examined. This was achieved by conducting a series of experiements in which young lambs were infected daily at a variety of infection rates with marked and unmarked worm populations.

Mathematical relations between factors and their impact on parasite regulation were formulated and parameters of these mathematical models estimated from the parasite data.

The data were consistent with simple model in which a threshold of worm exposure was required before resistance to incoming infective larvae developed. This was estimated to be a worm burden of 3532 worms. Once the threshold was exceeded the rate of development of resistance was independent of infection rate determined by host age alone. The development of resistance to new infection (establishment) was described by a logistic function in which the slope was a function of host age. The ET[50] (estimated time to reduce establishement by $50 \%$ after infection commenced) was a function of host age and the time taken to exceed the threshold.

While establishment was declining to low levels, adult worms accumulated without loss or minimal loss. When establishment was reduced to approximately $1 \%$ of incoming infective larvae, rejection of adult worms commenced and took approximately 10 weeks

Received 12th November, 1992.

Thesis submitted to the University of Technology, October 1991. Degree approved: August 1992. Supervisors: Professor A.G. Shannon and Dr. A.D. Donald.

Copyright Clearance Centre, Inc. Serial-fee code: 0004-9729/93 \$A2.00+0.00. 
to complete. However, this phase was characterised by marked heterogeneity in the timing of this host response.

Maximum egg production was estimated to be 780 eggs per adult female worm per day. However, fecundity decayed exponentially from this maximum value after the threshold worm burden was exceeded.

Most animals in these experiments were found to have a small population of worms whose development had been arrested at the exsheathed infective larval stage. Arrested development was described by a piecewise linear model as a function of host age and establishment rate.

The length of adult female worms was measured as they aged. This revealed a reduction in the size of the adult population. This was in part attributed to shrinkage of established adults rather than replacement of large adults by smaller worms.

To predict worm populations over time the above models were coupled together and worm numbers in each stage were updated daily. Predictions from this model were tested against data from a series of independent experiments in which host immune responses were induced by vaccination with irradiated larvae. Predictions of egg counts from independent experiements using daily infections were also made. In both cases the model gave good predicitons of the observed data.

Division of Animal Health

CSIRO

Private Bag I

PO Gelbe NSW 2037

Australia 